\title{
Estudio textural, mineralógico y metales asociados de la veta Julio Verne, depósito de Organullo, Puna salteña
}

D.o.I.: https://doi.org/10.30550/j.agl/2018.30.1/3

\author{
Natalia, SALADO PAZ ${ }^{1}$, Pamela A. TORRES CORREJIDOR ${ }^{2}$, \\ Valmir SOUZA DA SILVA ${ }^{3}$ \\ 1 Instituto de Bio y GeoCiencias del NOA (IBIGEO-UNSA-CONICET). Universidad Nacional de Salta, Fa- \\ cultad de Ciencias Naturales, Av. Bolivia 5150, CP 4000. Salta. \\ Mail: nataliasaladopaz@yahoo.com.ar \\ 2 Facultad de Ciencias Naturales, Universidad Nacional de Salta. Av. Bolivia 5150, CP 4000. Salta. \\ 3 nstituto de Geociencias. Universidad de Brasilia. Campus Universitário Darcy Ribeiro, Brasília. CEP \\ $70910-900$
}

> Resumen - El depósito Organullo (Pb-Cu-Zn-Ag-Au) se encuentra ubicado en la provincia geológica de la Puna (24ํ 24’ S], provincia de Salta. Corresponde a un sistema epitermal con dos importantes minas: Mina Julio Verne y Mina Torca, actualmente abandonadas con escombreras que contienen altas cantidades de mineral. La mineralización ocurre en vetas (Julio Verne, Diana, Natalia, Belén, Carolina, Marta, Virginia) alojadas en rocas de edades neoproterozoicas y ordovícicas. La veta de Julio Verne, objeto de este estudio, tiene rumbo NNO, de 200 metros de longitud y entre 0,2 y 0,4 metros de espesor. Constituye vetas controladas por fracturas hospedadas en la Formación Puncoviscana.

La compleja y amplia mineralogía de mena ya ha sido estudiada y asociada a sectores de las vetas pero no a las texturas minerales. En este trabajo se describen cinco texturas, (masiva, pseudobandeada, vetillas, brechosa y vuggyl asociadas a la mineralización. Se redefine ópticamente la presencia de pirita, calcopirita, bismutinita, covelina, luzonita-famatinita. Los microanálisis encristales de luzonita-famatinita permitieron caracterizar variaciones composicionales. El mapa de metales realizado por microscopia de barrido de alta resolución, muestra metales tales como $\mathrm{Sb}, \mathrm{As}, \mathrm{Cu}, \mathrm{Bi}, \mathrm{Pb}$ y $\mathrm{Sn}$ asociados a las texturas de brechas. El resultado del estudio de las texturas y la química de los minerales permite sugerir un depósitocomplejo, por lo que se deben realizar estudios de detalle a futuro para definir el tipo de depósito epitermal.

Palabras clave: Texturas, metales, epitermal, Puna.

Abstract - "Textural, mineralogical and associate metals study from Julio Verne mine, Organullo Deposit, Puna". The Organullo deposit ( $\mathrm{Pb}-\mathrm{Cu}-\mathrm{Zn}-\mathrm{Ag}-\mathrm{Au}$ ) is located in the geological province of the Puna (24 24' S), province of Salta. It corresponds to an epithermal system with two important mines: Julio Verne and Torca Mine, currently abandoned with slagheap that contain high amounts of ore. The mineralization occurs as veins (Julio Verne, Diana, Natalia, Belén, Carolina, Marta, Virginia) hosted in Neoproterozoic and Ordovician rocks. The Julio Verne vein, object of this study, has NNO strike, of 200 meters in length and between

\footnotetext{
> Ref. bibliográfica: Salado Paz, N.; Torres Correjidor, P. A.; Souza Da Silva, V. 2018. Estudio textural, mineralógico y metales asociados de la veta Julio Verne, depósito de Organullo, Puna salteña. Acta geológica lilloana 30 (1): 31-45.

> Recibido: 19/01/18 - Aceptado: 29/06/18

> URL de la revista: http://actageologica.lillo.org.ar

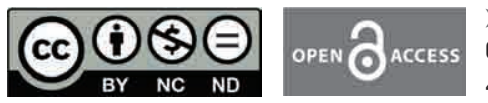

> Algunos derechos reservados. Esta obra está bajo una Licencia Creative Commons Atribución - No Comercial - Sin Obra Derivada 4.0 Internacional.
} 
0.2 to 0.4 meters thick. It constitutes vetiform structures controlled by fractures hosted in the Puncoviscana Formation.

The complex and extensive mineralogy of ore has already been studied and associated with sectors of the vetiforms but notto the mineral textures. In this work, we observe five textures (massive, pseudobanded, veinlets, vuggy and breccia) associated with the mineralization. The presence of pyrite, chalcopyrite, bismutinite, covelline, luzonite-famatinite is optically redefined. The microanalyses of luzonite-famatinite crystals allow the characterization of compositional variations. The metal map made by high resolution scanning electron microscopy shows metals such as $\mathrm{Sb}, \mathrm{As}, \mathrm{Cu}, \mathrm{Bi}, \mathrm{Pb}$ and $\mathrm{Sn}$ associated with breccias textures. The study of the textures and minerals suggests a complex system, therefore, detailed studies should be carried out in the future for to define the type of epithermal system.

Keywords: Texture, metals, epithermal, Puna.

\section{INTRODUCCIÓN}

El depósito Organullo (Pb-Cu-Zn-Ag-Au) se encuentra ubicado en la provincia geoló-

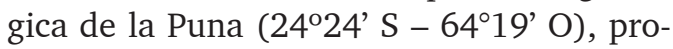
vincia de Salta, a aproximadamente $20 \mathrm{~km}$ al Sur de la localidad de San Antonio de los Cobres. Posee dos importantes minas: Mina Julio Verne y Mina Torca que han sido explotadas en 1930. Las vetas de Julio Verne se explotaron en una extensión de $160 \mathrm{~m}$, por medio de un cortavetas, dos galerías subterráneas y tres piques para beneficio de oro, plata, cobre y bismuto, con valores medios de $4 \mathrm{~g} / \mathrm{t}$ de Au (Sureda et al. 1994). Posteriormente se explotó Au aluvional, de manera rudimentaria, en el margen del río Organullo. La empresa Cardero Resource Corporation, en exploraciones realizadas en el año 2011, determinan mediante pozos valores de Ag (máximo 4,196 ppm-mínimo 0,302 ppm) y Au (máximo 0,957 ppm-mínimo 0,142 ppm). Los últimos trabajos exploratorios de la empresa Artha Resources Corporation (2012) mencionan altos tenores de $\mathrm{Cu}-\mathrm{Bi}$, y tenores de $\mathrm{Au}$ de hasta $37 \mathrm{~g} / \mathrm{t}$ asociado a la pirita. Actualmente el depósito se encuentra abandonado con escombreras que contienen altas cantidades de mineral.

Desde su descubrimiento hasta la actualidad, se han realizado aportes sobre la mineralogía de mena y alteración hidrotermal (Viera 1975, Argañaraz 1980, Sureda et al. 1994, Fonseca 2009, Torres Correjidor et al.2016), vinculando la mineralización a la actividad volcánica del cuerpo dómico de Organullo (Viera 1975, Ramallo et al. 2011).
El depósito ha sido clasificado inicialmente como epitermal de baja sulfuración con superposición de alta sulfuración (Klipfel 2009) y posteriormente como de alta sulfuracióncon transición apórfido(Cardero Resources Report 2011).

Los minerales de mena han sido estudiados y determinados por Paar et al. (2000a, 2000b) y Paar et al. (2010) quienesestablecieron tres etapas de mineralización. La primera etapa comprende la formación de abundante pirita, casiterita y miembros de la serie estannita-kesterita. Te -canfieldita puede ocurrir en esta etapa en asociación con abundante matildita y sulfosales de Ag-Pb-Bi (benjaminita). El proceso de mineralización continuó con una segunda etapa durante la cual se cristalizaron las sulfosales de $\mathrm{Bi}$ (bismutinita, aikinita, serie emplectita-calcoestibita, hodrushita), seguidos por tetraedrita-tennantita (localmente Bi enriquecido), enargita (posible luzonita-famatinita), especies que contienen Sn (mohita, kuramita), trazas de kawazulita y oro nativo. Tetradimita ocurre como granos pequeños en la matriz tenantífera. La etapa final está representada por pequeñas cantidades de bornita (con calcopirita) y especies de Sn (mawsonita, vinciennita, stannoidita). También sugieren que digenita y covelina, serían minerales formados por supergénesis.

Si bien los estudios mineralógicos previos definen las paragénesis minerales, no han identificado las texturas de mineralización asociadas a los eventos.

En este trabajo, describimos las texturas observadasen la veta Julio Verne y aporta- 
mos información sobre su mineralogía y posible génesis.

\section{METODOLOGÍA}

A partir del mapeo y muestro en trabajo de campo de la mineralización, se realizó un estudio textural macroscópico y bajo lupa binocular de muestras desuperficie. Se confeccionaron 19 láminas petrocalcográficas y 6 probetas calcográficas. Las secciones fueron realizadas en el laboratorio de Petrotomía y Mineralogía de la Universidad Nacional de Salta y laboratorio de Petrotomía de la Universidad Nacional de San Luis.

Se realizaron estudios petrográficos con microscopio óptico de polarización marca Olympus BX51 ubicado en la sala de microscopía de la Facultad de Ciencias Naturales, UNSa y con microscopio petrocalcográfico marca Zeiss ubicado en el Instituto de Bio y Geociencias del NOA, Rosario de Lerma.

Se efectuaron análisis de microsonda en 4 muestras de rocas mineralizadas. Los análisis químicos de los minerales, fueron llevados a cabo en los laboratorios del Instituto de Geociencias de la Universidad Federal de Brasilia. La microsonda es un equipo Jeol JXA8230, posee 5 espectrómetros de longitudes de onda (WDS) y un espectrómetro de dispersión de energías (EDS), con cristales analizadores (TAPJ, LIF, LIFH, PETJ, PETH, LDE1 e LDE2) que permiten la detección y análisis de todos los elementos químicos con número atómico superior a cuatro.

Se efectuaron dos mapas de metales en una muestra de mineralización, con el fin de determinar la presencia de metales diferentes a los observados en la mineralogía calcográfica y petro-calcográfica. Para ello se realizó el estudio sobre una lámina delgada pulida. El equipo usado es un microscopio electrónico de barrido de presión variable VPSEM modelo SUPRA 40VP del centro de Instrumentación Científica de Granada, España.

\section{GEOLOGÍA DEL ÁREA}

La zona de estudio se encuentra en la provincia geológica de la Puna donde el basamento está representado por la Formación Puncoviscana (Turner 1960) de edad Neoproterozoico - Cámbrico inferior (Aceñolaza y Aceñolaza 2005), constituido en la región de San Antonio de los Cobres, por secuencias sedimentarias de pelitas, areniscas, grauvacas y metacuarcitas afectadas por metamorfismo de bajo a medio grado que dio lugar a secuencias metasedimentarias de filitas, pizarras, grauvacas, esquistos y hornfels. Estas secuencias se encuentran altamente deformadas con diferentes estilos de plegamientos dependiendo de la competencia de la roca, y han sido interpretadas como depositadas en ambiente marino profundo (Omarini 1983) (figura 1).

El Paleozoico inferior está representado por una secuencia sedimentaria, denominada Formación Parcha (Harrington y Leanza 1957), constituida por pizarras, lutitas e intercalaciones de cuarcita portadoras de graptolitos del Tremadociano - Darriwiliano (Rolleri y Mingramm 1968). Intercalan en la secuencia sedimentaria, niveles hialoclásticos dacíticos asociados a flujos de lava (Coira y Koukharsky 1994) y en otros lava espilíticas intercaladas con turbiditas submarinas del Paleozoico inferior (Coira y Koukharsky 1991) que evidencian la presencia de un volcanismo submarino bimodal sin-sedimentario desarrollado durante el Ordovícico inferior. También se han descripto ignimbritas y tobas riodacíticas con pelitas (por ejemplo, Quebrada Tuzle, Sierra de Cobre) a lo que se los ha denominado Formación Agua de Castilla (Koukharsky y Mirré 1974). Recientemente (Quiroga 2014) describe intercalaciones de pelitas con vulcanitas coherentes, hialoclastitas y peperitas en el sector Quebrada de Cajón y Quebrada Tuzgle (Quiroga com. pers. 2017). Los afloramientos ordovícicos del área están siendo redefinidos en estudios actuales, por lo que en este trabajo denominamos a los afloramientos observados como «Ordovícico indiferenciado», haciendo referencia en donde aflora pelita y vulcanita intercalada con pelitas. La unidad en el área de estudio presenta una intensa fracturación y alteración lo que dificulta la distinción de los contactos entre las unidades 
formacionales definidas como Parcha y Agua de Castilla.

Cubren en parte esta secuencia proterozoica-paleozoica, sucesiones sedimentarias continentales correspondientes al Grupo Salta (Turner 1960) de edad Cretácica- Paleocena. Particularmente, el Subgrupo Pirgua (Cretácico) integrado principalmente por conglome-

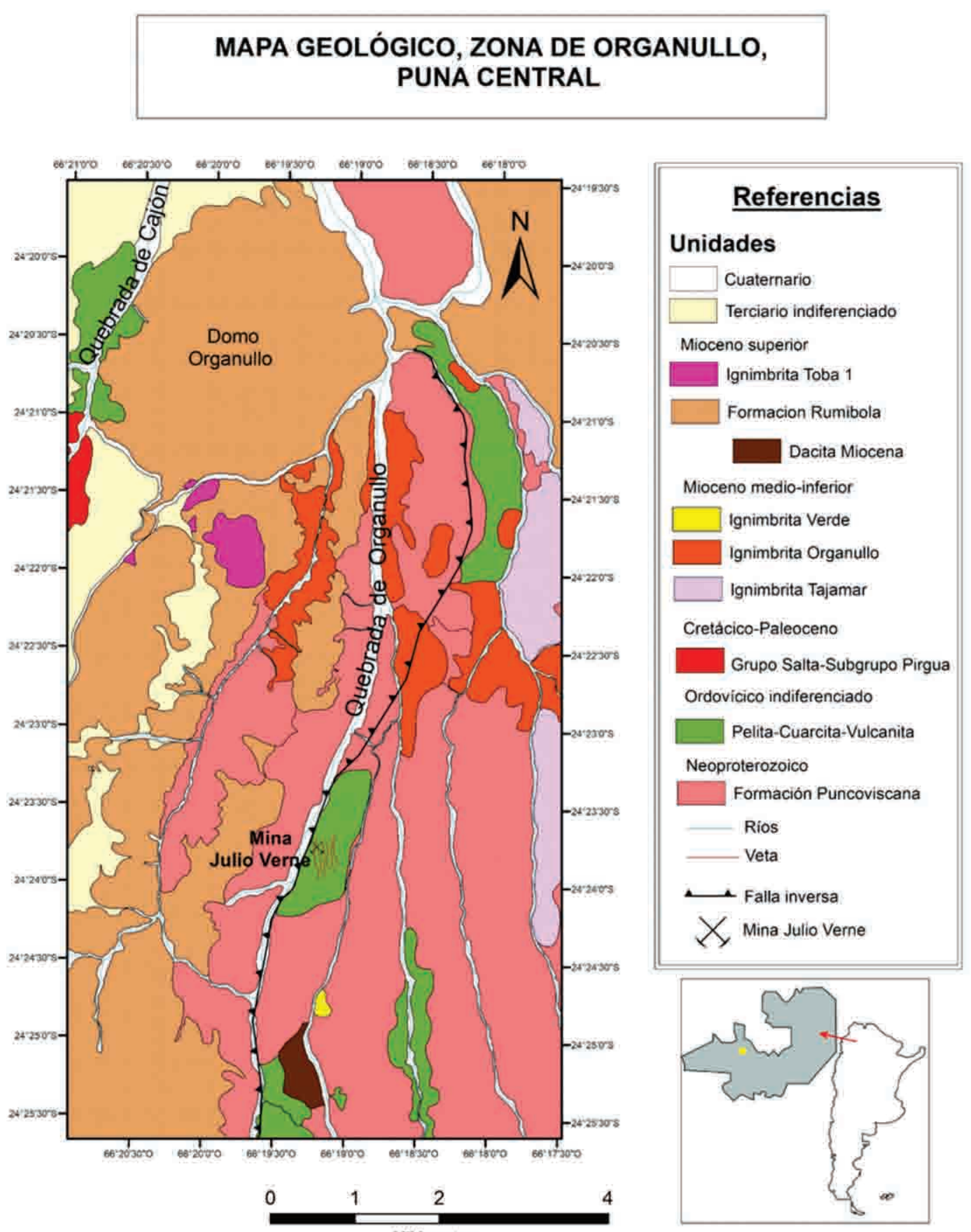

Figura 1. Mapa geológico de la zona de Organullo. 
rados, areniscas y fangolitas de colores rojos, rosados y grises amarillentos, es de unos 2000 metros de potencia (figura 1 ).

El registro sedimentario Terciario está representado por el Grupo Pastos Grandes (Turner 1960).En la zona de estudio, aflora en la Quebrada de Cajón cubriendo los afloramientos del Subgrupo Pirgua (Blasco et al. 1996). El Cenozoico se encuentra representado por depósitos ignimbríticos provenientes de la caldera de colapso Aguas Calientes
(Petrinovic et al. 2010), Ignimbrita Verde (equivalente a Ignimbrita Aguas Calientes (Coira y Paris 1981) e Ignimbrita Tajamar (equivalente a Ignimbrita Abra del Gallo (Coira y Paris 1981).

La actividad volcánica miocena característica del área está representada por el domo subvolcánico Organullo,de edades que oscilan entre los 12,1 y 13,5 Ma ( $\mathrm{Ja}$ pan International Cooperation Agency 1993, Petrinovic et al. 1999). Está compuesto de

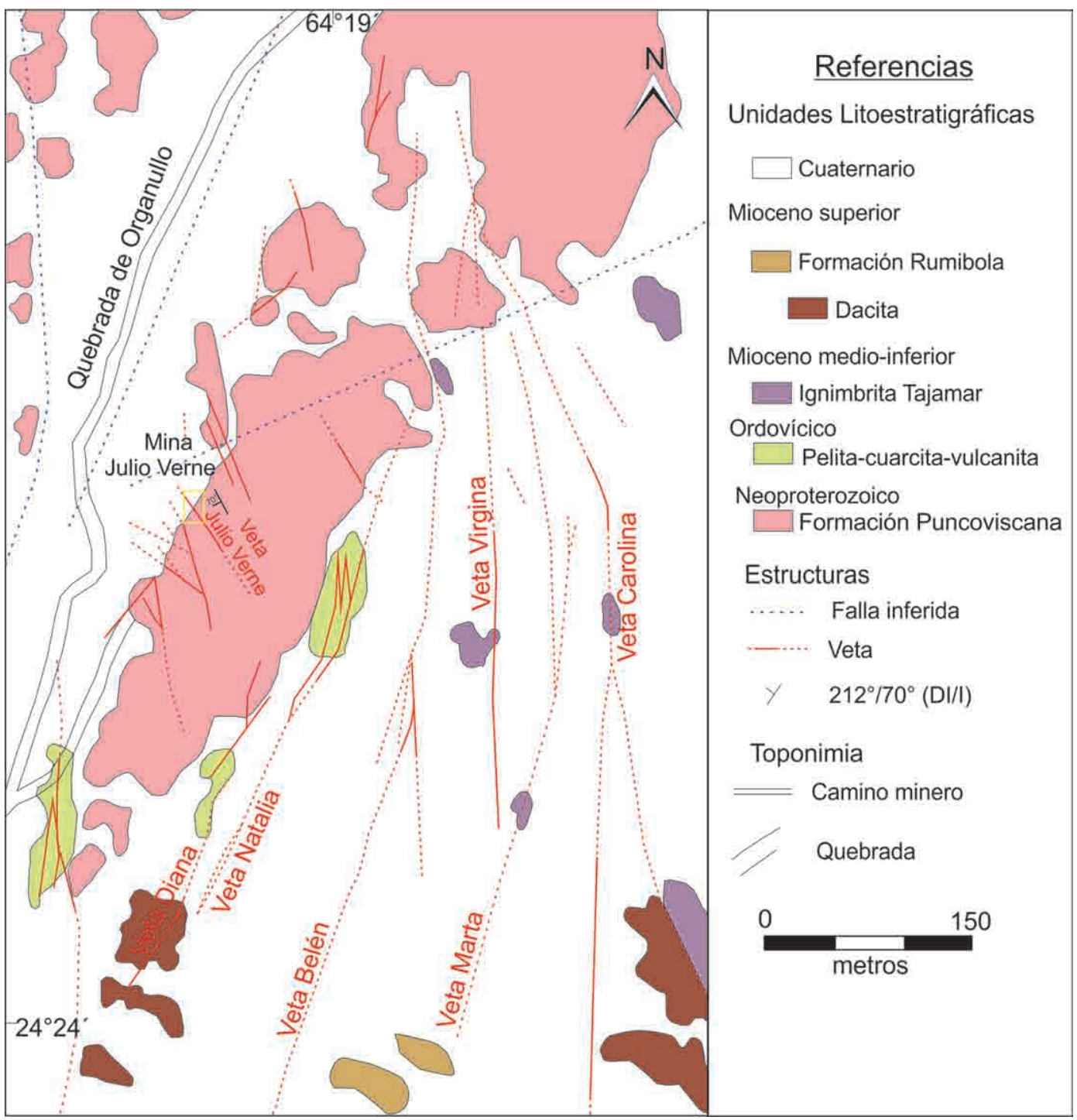

Figura 2. Mapa de la mineralización modificada de Artha Reources Corporation (2012), mostrando la geología, estructura vetiforme y datos estructurales tomados en el campo. En recuadro amarillo se muestra la veta estudiada en este trabajo. 
coladas dómicas de composición dacítica y depósitos de bloque y cenizas asociados (Formación Rumibola). Cubren parcialmente al domo, afloramientos de la Ignimbrita Organullo de 13-12 Ma (Hidroproyectos 1985 y Petrinovic 1989), correlacionable con la Ignimbrita Vizcachayoc definida por Ramos (1973) e ignimbrita Toba 1 de 7,4 Ma definida por Petrinovic et al. (1999) en la zona del cuerpo dómico El Morro.

Recientemente (Torres Correjidor, 2017) se ha definido una unidad, Dacita Miocena, constituida por un cuerpo de $500 \mathrm{~m}$ de espesor, que por las relaciones estratigráficas podría corresponder al Mioceno.

\section{RESULTADOS}

La veta de Mina Julio Verne, aflora en el margen oriental de la quebrada de río Org- anullo. Corresponde a un sistema de vetas (Julio Verne, Diana, Natalia, Belén, Carolina, Marta, Virginia) de rumbo N-S y NNO-SSE (figura 2). Las rocas de caja corresponden a metapelitas de la Formación Puncoviscana y rocas del Ordovícico que se encuentran intensamente alteradas. La «veta» Julio Verne, poseerumbo NNO (predominante $\mathrm{N} 330^{\circ} \mathrm{O}$, $\mathrm{N} 302^{\circ} \mathrm{O}$ ), con aproximadamente 120 metros de longitud y entre 0,2 a 0,4 metros de espesor. A escala de afloramiento se observa que las mineralizaciones corresponden principalmente a vetas, con baja a moderada proporción de mineral diseminado alojados en la Formación Puncoviscana (figura 3).

\section{TEXTURAS DE LA MINERALIZACIÓN}

Las texturas son:

1) Pseudobandeadas: la observación ma-

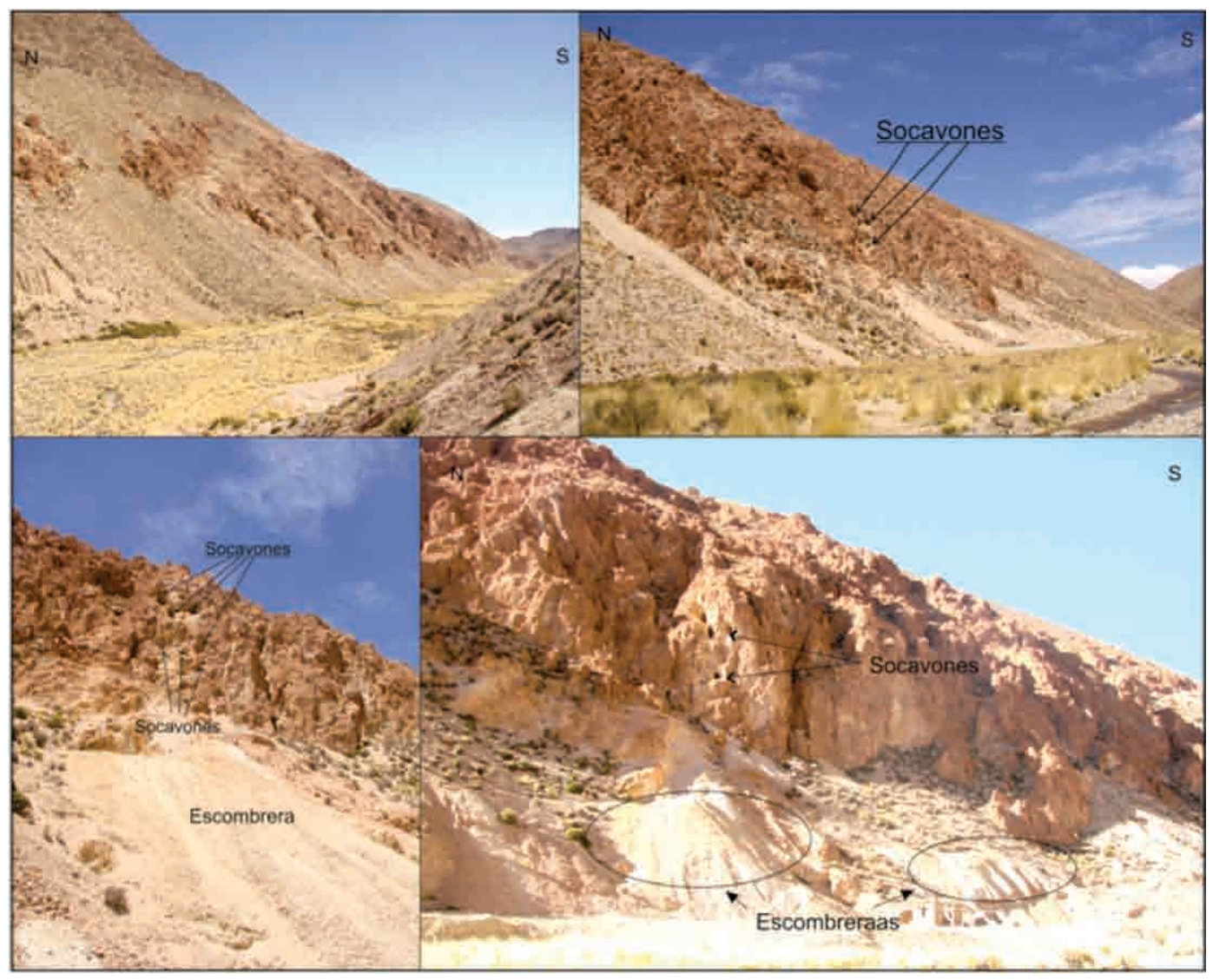

Figura 3. Fotografía de afloramientos por la Quebrada de Organullo donde se puede visualizar los laboreos mineros (socavones) y las escombreras. 


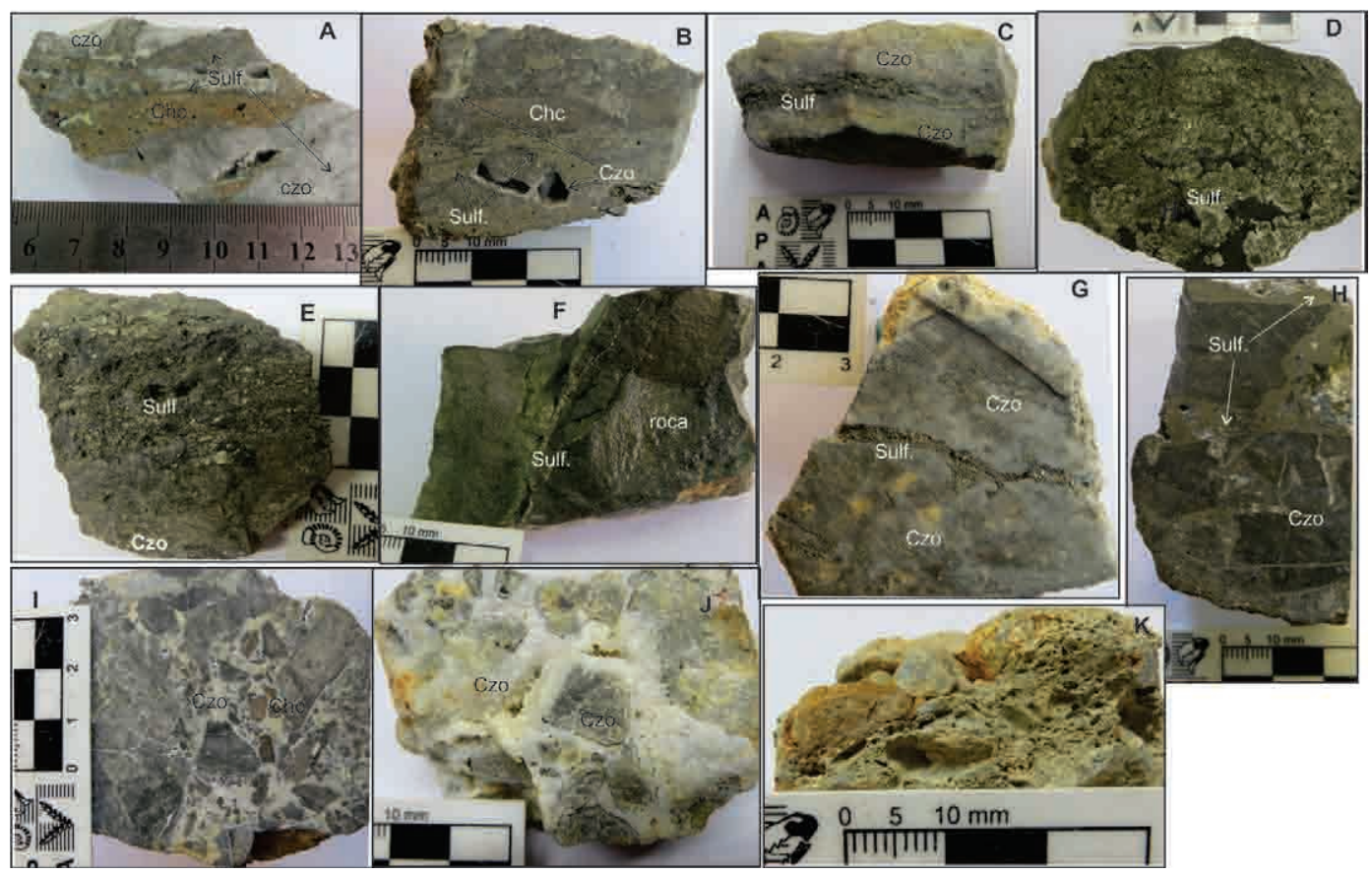

Figura 4. Fotografías de muestras de mano de la veta. En A, By C) texturas pseudo bandeadas compuestas de cuarzo, calcedonia y sulfuros. En D y E) Texturas masivas compuestas en su totalidad de sulfuros en una matriz de cuarzo fino. En F, G y H) texturas en vetillas de sulfuros en una matriz cuarzosa. En I y J] texturas brechosas. En I] textura brechosa constituida por venas de calcedonia que incorpora fragmentos del vetiforme en clastos angulosos, no rotados. En J] textura brechosa constituida por clastos subangulosos a redondeados de cuarzo con sulfuros (Fragmentos delas vetas) con cemento de cuarzo cristalino, por sectores en geodas. En K) textura vuggy en cuarzo. Referencias: Qz: cuarzo; sulf: Sulfuro (pirita y calcopirita); chc: Calcedonia.

croscópica permite definir bandas de sulfuros (pirita y calcopirita) y bandas de cuarzo y/o calcedonia. Las bandas de calcedonia son rosadas, con un espesor variable de 1,5 a 0,5 $\mathrm{cm}$, no paralelos e irregulares, que intercalan con finas bandas de sulfuros (menor a $1 \mathrm{~mm}$ ) y bandas de $2,5 \mathrm{~cm}$ de cuarzo cristalino de grano fino (figura 4 A-B). También se observan variaciones de esta textura donde no hay bandas de calcedonia, pero sí de cuarzo alternadas con bandas de sulfuros (figura 4C) y con cuarzo con abundantes sulfuros. Esta textura no está muy desarrollada.

Bajo lupa binocular se observa que las texturas bandeadas presentan sulfuros en bandas y en cristales diseminados dentro de cuarzo y calcedonia rosa. Las bandas son finas, son las más abundantes con espesores de $1 \mathrm{~mm}$, que se localizan entre las bandas de cuarzo y calcedonia. También se observa que los sulfuros constituyen cristales anhedrales a subhedrales inmersos de manera diseminada en cuarzo y en calcedonia (figura 5 A-B-C).

2) Formas masivas: aglomerados de sulfuros (pirita y calcopirita) que constituyen masas de hasta $5 \mathrm{~cm}$ en muestras de mano. Esta textura en general está compuesta en su totalidad por sulfuros puros masivos, aunque también se han observado abundantes sulfuros en una masa fina de cuarzo. A pesar de la abundancia de los sulfuros, los granos individuales presentan tamaños menores a los $5 \mathrm{~mm}$, por lo que no es posible determinar otros minerales (aparte de pirita y calcopirita) macroscópica (figura 4D-E). Esta textura es la más representativa en el área.

3) Vetillas: constituyen el relleno de frac- 

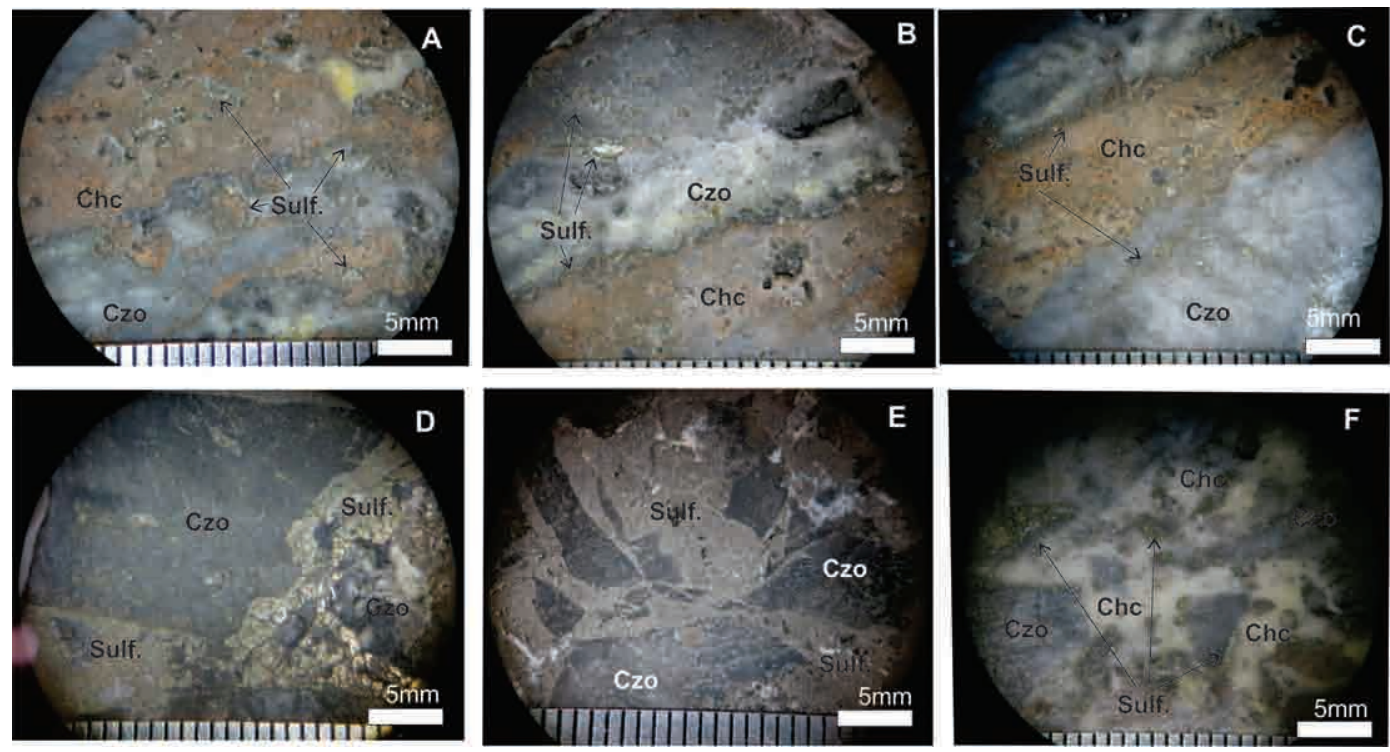

Figura 5. Fotografías de las texturas bajo lupa binocular. A, B y C) veta de textura bandeada compuesta de calcedonia, cuarzo y sulfuros. Puede observarse que los sulfuros principalmente componen bandas finas entre cuarzo y calcedonia rosa y también se presentan en cristales diseminados en cuarzo y calcedonia. D y E] veta de textura en vetillas compuesta de cuarzo fino con vetillas de sulfuros. Puede observarse que las vetillas son irregulares, acompañadas de escaso cuarzo en algunos sectores. FJ veta de textura brechosa, donde se observa un sector de la matriz calcedónica con sulfuros que se presentan tanto en la matriz como en los clastos. Referencias: Qz: cuarzo; Chc: calcedonia; Sulf.: sulfuros.

turasen las rocas de caja: pelitas de la Formación Puncoviscana (figura 4 F) y en la cuarcita del Ordovícico (figura 4 G). No presentan una orientación preferencial. Corresponden a vetillas de sulfuros (principalmente pirita y calcopirita) con espesores variables de $0,5 \mathrm{~cm}$ a $2,5 \mathrm{~cm}$. Las vetillas de sulfuros también se desarrollan en los sectores más próximos al contacto con la vetas con espesores mayores de $2,5 \mathrm{~cm}$ (figura $4 \mathrm{H}$ ).

Bajo lupa binocular se observan los sulfuros constituyendo vetillas irregulares con cristales de sulfuros (figura $5 \mathrm{D}$ ) y vetillas de abundantes sulfuros de grano fino (figura 5 E). Se observa, además la presencia escasa de un cuarzo blanco que aparece por sectores junto a las vetillas de sulfuros. Cabe destacar que el cuarzo que contiene las vetillas también presenta sulfuros diseminados de grano fino, menor a $1 \mathrm{~mm}$ (figura 5D y E).

4) Brechosa: se observan 2 brechas monomícticas constituidas por clastos de las mineralizacionesde textura masiva cementados por calcedonia. La brecha 1 constituida por clastos angulosos, con tamaños de 0,2 a 1,5 $\mathrm{cm}$ con texturas de puzzle no rotados características de brechas hidrotermales, en una matriz de calcedonia blanca (figura 4I). La brecha 2 constituida por clastos subangulosos a redondeados de fragmentos de mineralización de textura masiva, de tamaños de 1,5 a $3,5 \mathrm{~cm}$, inmersos en una matriz compuesta por cuarzo cristalino blanco, con algunas texturas típicas de relleno de espacios abiertos tales como geodas y crustiforme (figura 4J).

Bajo lupa binocular se observa la textura brechosa compuesta de clastos angulosos en cemento de calcedonia blanca.Los sulfuros (pirita y calcopirita) se concentran principalmente entre el contacto del cemento y clastos y en los clastos. El cemento se compone de finos cristales de sulfuros de escala micrométrica (figura 5F).

En todos los casos, los sulfuros son de grano fino (en general, menores al $\mathrm{mm}$ pero 
Tabla 1. Composiciones minerales para las muestras estudiadas.

\begin{tabular}{|c|c|c|c|c|c|c|c|c|c|c|c|c|c|c|}
\hline \multirow[b]{2}{*}{ Muestras } & \multirow[b]{2}{*}{ Minerales } & \multicolumn{7}{|c|}{ Valores en $W t \%$} & \multicolumn{6}{|c|}{ Formulas en A. P.U. F } \\
\hline & & $\mathrm{S}$ & $\mathrm{Fe}$ & $\mathrm{Cu}$ & As & $\mathrm{Sb}$ & $\mathrm{Bi}$ & Total & $\mathrm{S}$ & $\mathrm{Fe}$ & $\mathrm{Cu}$ & As & $\mathrm{Sb}$ & $\mathrm{Bi}$ \\
\hline $\begin{array}{l}\text { E1D, EV13A, EV13B, } \\
\text { E1B }\end{array}$ & Pirita $(n: 46)$ & 53,32 & 46,58 & 0,33 & & & & 100,23 & 2 & 1 & 0,01 & & & \\
\hline E1D, EV13A, EV13B & Calcopirita (n:10) & 34,63 & 29,27 & 34,9 & & & & 99,02 & 2 & 0,97 & 1,02 & & & \\
\hline E1D, EV13A, EV13B & Famatinita (n:23) & 29,38 & & 44,48 & 1,35 & 24,03 & & 99,26 & 4 & & 3,06 & 0,08 & 0,86 & \\
\hline E1D & Luzonita (n:9) & 27,43 & 6,839 & 43,1 & 17,79 & 5,1 & & 100,27 & 4 & 0,57 & 3,17 & 1,11 & 0,2 & \\
\hline EV13B, E1B & Bismutina ( $\mathrm{n}: 8)$ & 19,22 & & & & 1,37 & 79,73 & 100,32 & 1,89 & & & & 0,06 & 3 \\
\hline E1D, EV13A & Covelina (n:4) & 32,3 & 4,36 & 62,96 & & & & 99,63 & 1 & 0,08 & 0,98 & & & \\
\hline
\end{tabular}

algunos menores al $\mathrm{cm}$ ) lo que impide su clasificación a muestra de mano y lupa binocular. Todos los sulfuros poseen coloraciones amarillentas y verdosas, y en menor medida, coloraciones grises.

5) Vuggy: si bien esta textura no es abundante, se la ha observado en fragmentos de la escombrera. Presenta oquedades de diferentes tamaños en una matriz de cuarzo cristalino junto a cristales de sulfuros de tamaño menor al mm (figura $4 \mathrm{~K}$ ).

\section{MiNERALOGÍA Y MAPA DE METALES}

Los minerales de mena son de grano fino y medio (menor a $0,5 \mathrm{~mm}$ ) y se encuentran presentes en las texturas previamente descriptas. Principalmente se distinguiopirita, calcopirita, famatinita-luzonita y bismutina, tetraedrita-tenantita, covellina.

La pirita es el sulfuro más abundante, se presenta en granos subhedrales a euhedrales de tamaños promedios de 0,5 $\mathrm{mm}$ (figura 6 A, C y E). La calcopirita, es anhedral, con tamaños de hasta $0,7 \mathrm{~mm}$ (figura $6 \mathrm{~A}, \mathrm{~B}$ y C). La bismutina, se presenta en forma de cristales subhedrales con hábitos tabulares de hasta $3 \mathrm{~mm}$ (figura $6 \mathrm{D}$ ). La famatinita se presenta en cristales anhedrales de hasta $1 \mathrm{~mm}$ (figura $6 \mathrm{~A}$, B y C). La luzonita constituye pequeños cristales anhedrales de hasta $0,5 \mathrm{~mm}$, como inclusiones de cristales de pirita y de calcopirita(figura 6 B). La tenantita-tetraedrita se presenta como cristales anhedrales de hasta $1 \mathrm{~mm}$, principalmente asociada a la pirita (figura 6E).

La covelina se presenta en cristales anhedrales y como alteración en los bordes de calcopirita con tamaños de $0,2 \mathrm{~mm}$ (figura 6 F). A partir de los datos de microanálisis obtenidos por microsonda electrónica para famatinitay luzonita, se graficó un diagrama triangular, donde los vértices corresponden al $100 \%$ de As, Sb y Cu, para observar las variaciones composicionales entre famatinita $\left(\mathrm{Cu}_{3} \mathrm{SbS}_{4}\right)$ y luzonita $\left(\mathrm{Cu}_{3} \mathrm{AsS}_{4}\right)$ con respecto a composiciones ideales de estos minerales según Gaines (1957). En la figura 7 también se ploteó valores químicos obtenidos de los antecedentes (Paar et al. 2010) para estos minerales. Se observa que los valores obtenidos en este trabajo, muestran mayor dispersión entre el $\mathrm{Sb}$ y el As, con muy poca dispersión en Cu. Sin embargo se encuentran en el rango de contenidos para la solución sólida. Algunos microanálisis se encuentran muy próximos a las composiciones ideales de Gaines (1957). Los valores químicos obtenidos por Paar et al. (2010) para famatinita y luzonita son más homogéneos y con poca dispersión ya que corresponden a un único cristal. También se determinó mediante microsonda electrónica bismutinita, pirita, calcopirita y covelina (Tabla1).

Con el fin de determinar la mineralogía de grano fino a muy fino se realizaron mapas de metales en una muestra dela veta (figura 8). La muestra corresponde a la zona de mineralización con textura brechosa, donde se analizó un sector de un clasto (compuesto por cuarzo y sulfuros). Sobre la misma área se mapearon $\mathrm{As}, \mathrm{Cu}, \mathrm{Bi}, \mathrm{Sn}, \mathrm{Pb}$ y Sb. Se observa que $\mathrm{Sb}$, As y Bi presentan incrementos en las mismas áreas, lo que podría indicar minerales de estos metales en grano muy fino. Mientras que Bi también está asociado a incrementos en $\mathrm{Pb}$. El Cu incrementa levemente en la parte central de la imagen (punto 3 en la imagen BSE), aunque está ausente en el resto de la imagen. El Sn se concentra formando cristalesde casiterita(figura8). Los 

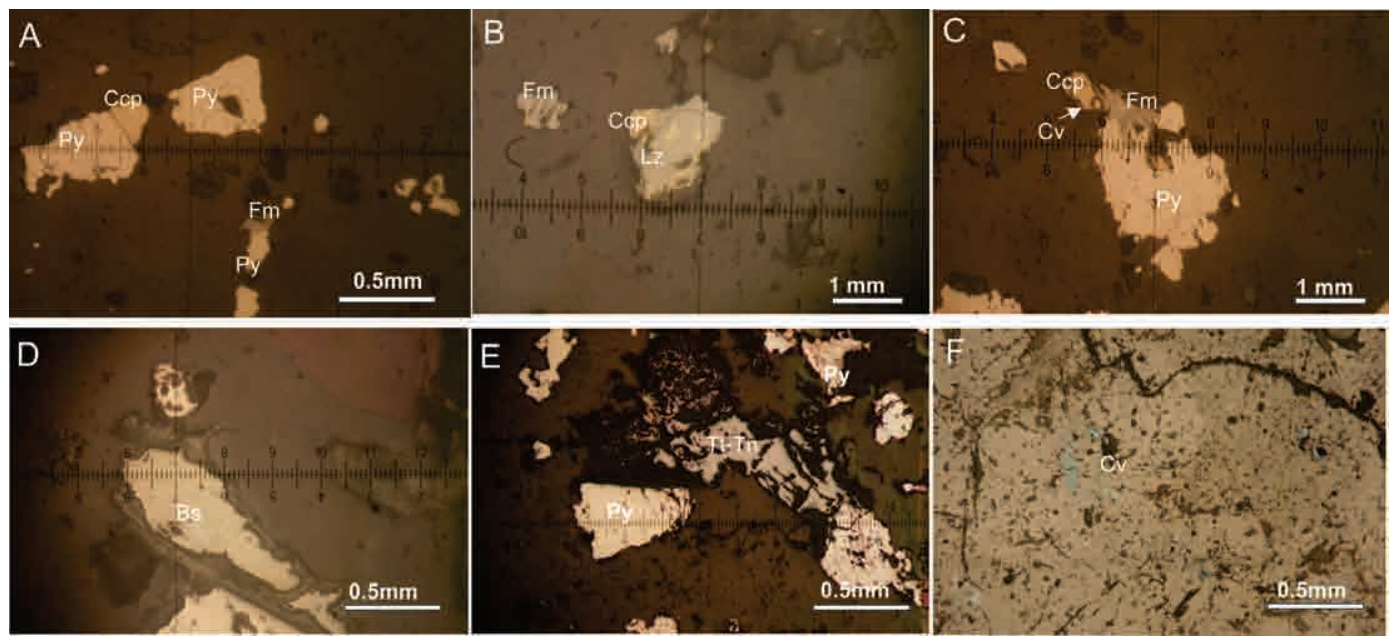

Figura 6. Fotomicrografía - A] Cristal de pirita y cristales de calcopirita con famatinita. Aumento 10X. B) Agregado de famatinita y pirita. Aumento 20X. C) Agregado de pirita, con famatinita y calcopirita alterada en los bordes a covelina. Aumento 20X. D) Cristal de bismutina a 10X. E) Cristales de pirita y tenantita-tetraedrita. Aumento 10x. F) Cristales de covelina. Aumento 40X. Referencias: Py: pirita; Ccp: calcopirita; Fm: famatinita; Bsm: bismutinita; Lz: Iuzonita; Sp: esfalerita; Cv: covelina.

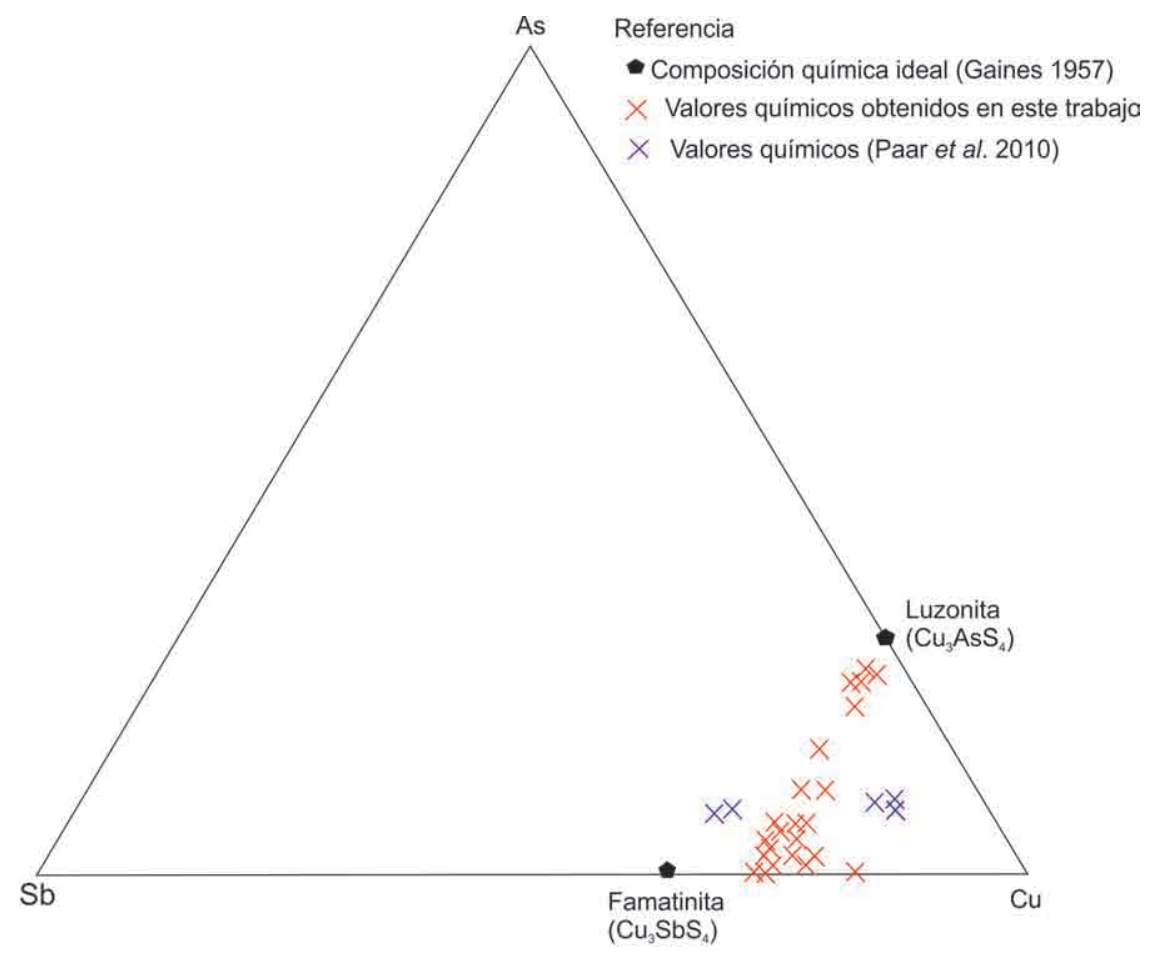

Figura 7. Diagrama triangular entre As-Sb-Cu, mostrando los valores químicos ideales determinados por Gaines (1957), para los sulfuros de luzonita $\left(\mathrm{Cu}_{3} A s S_{4}\right)$ y famatinita $\left(\mathrm{Cu}_{3} \mathrm{SbS}_{4}\right]$, los valores químicos obtenidos en este trabajo $[X]$ y valores químicos obtenidos por Paar et al. (2010) (X), para luzonita y famatinita. 

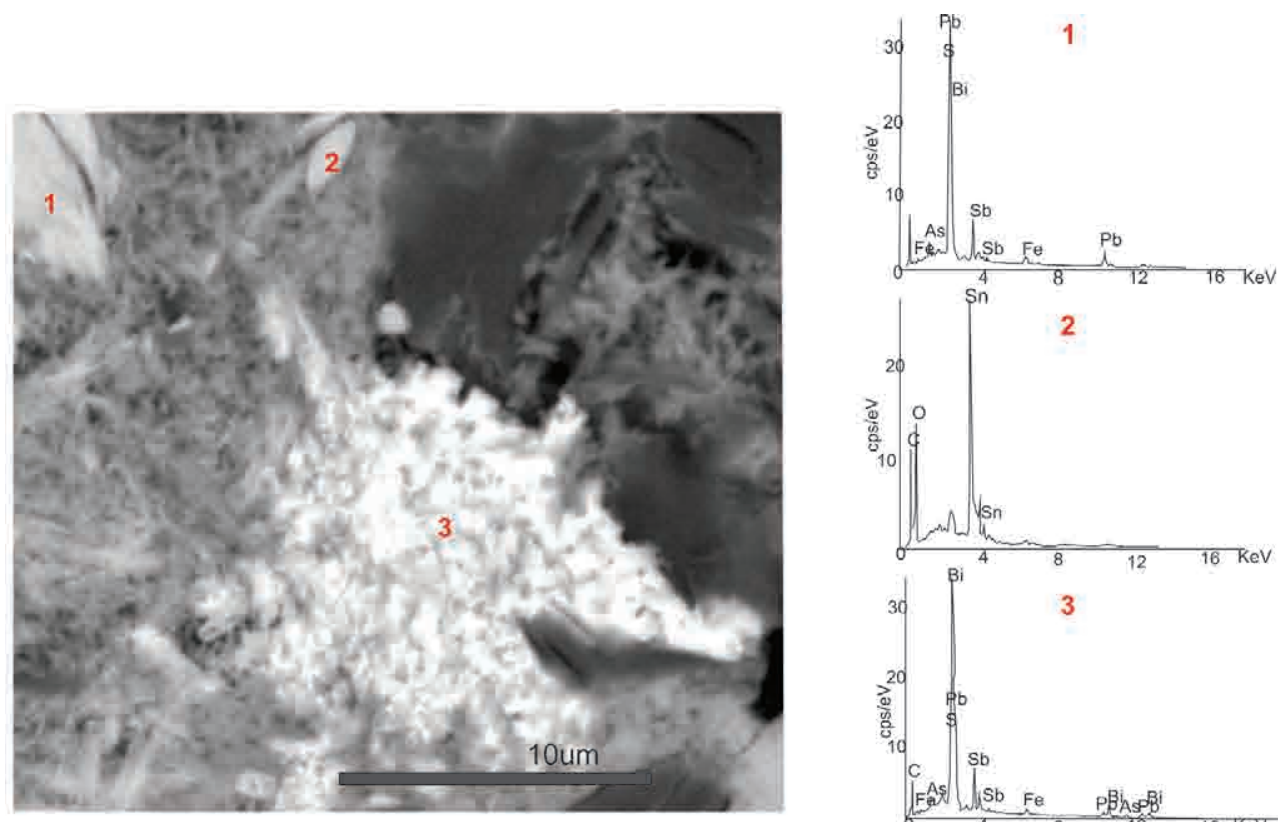

CuK

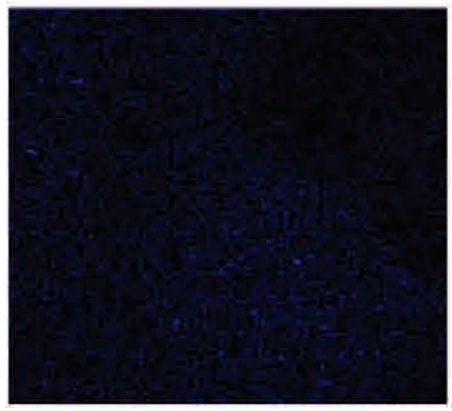

$\mathrm{Sb} \mathrm{L}$
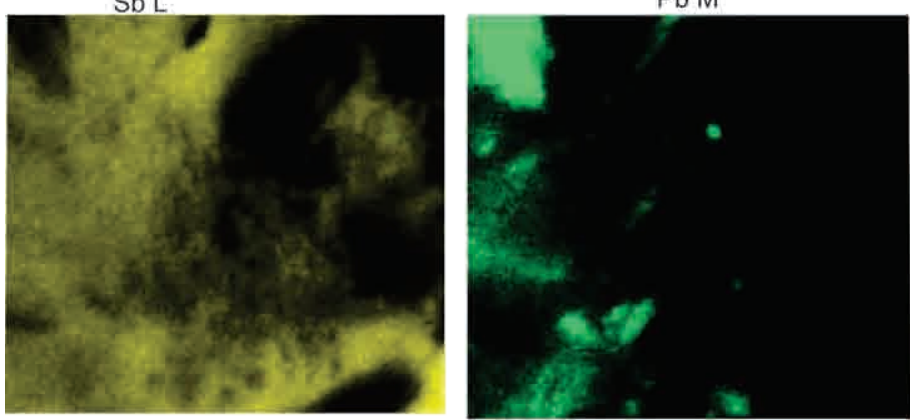

$\mathrm{Pb} \mathrm{M}$
2
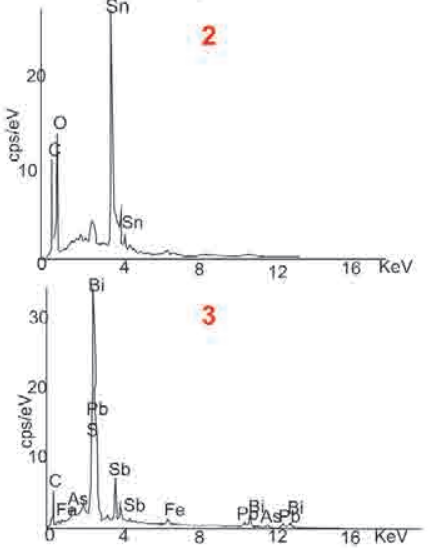

$\mathrm{SnK}$
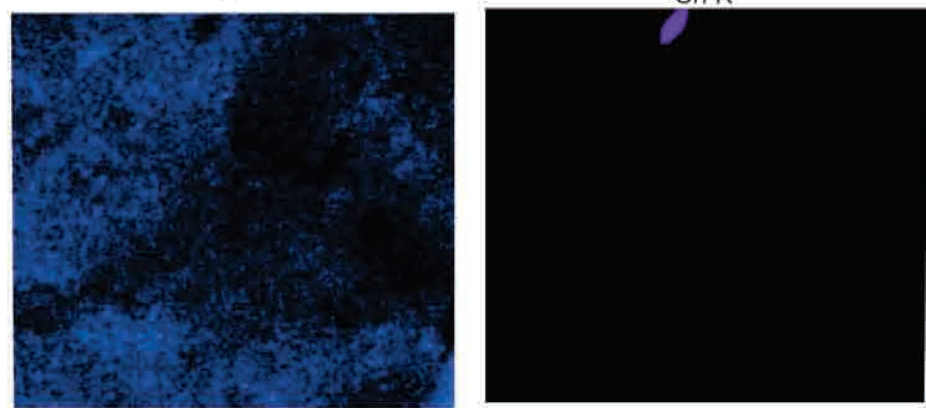

BiM

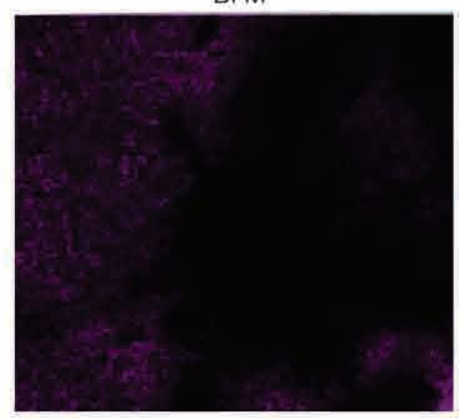

$10 \mathrm{um}$

Figura 8. Imagen BSE de un sector de la muestra EA1 con textura brechosa. Análisis cualitativo y mapas de variaciones de metales. 
espectros cualitativos no permiten identificar con precisión las proporciones atómicas para determinar fases minerales, excepto para la casiterita.

\section{DISCUSIÓN}

Inicialmente, la Mina Julio Verne fue definida como un yacimiento de tipo epitermal por Viera (1975) y Argañaraz (1980). La mineralogía de mena primaria constituida por galena, esfalerita, pirita, calcopirita, tenantita, tetraedrita, bismutina, bornita (Viera 1975 y Argañaraz 1980) correspondería a un sistema de baja sulfuración y sulfuración intermedia según las clasificaciones de Hayba et al. (1985), Heald et al. (1987), Hedenquist (1987) y Hedenquist et al. (2000). Sin embargo, el modelo de mineralización del depósito de Organullo está en discusión por diferentes autores (Klipfer 2009, Cardero Resources Report 2011, Artha Resources 2012) quienes plantean diferentes modelos (epitermal de baja sulfuración, de alta sulfuración, sistema porfídico y/o transiciones entre ellos). El estudio más completo (Klipfel 2009) considera que la alteración y mineralización observada en la zona Organullo, puede ser parte de un sistema de salida hidrotermal con características de sulfuración baja y alta.

En este trabajo, se observó que las diferentes texturas no muestran variaciones representativas a escala de afloramiento. En general, se observa que las texturas masivas son las más abundantes, componen el centro de las estructuras vetiformes y por sectores gradúan a texturas pseudobandeadas, y luego a texturas en vetillas en las rocas de caja. Las texturas en brechas no son abundantes, representa un estadio posterior a las anteriores, ya que incorpora los fragmentos mineralizados de las texturas masivas como clastos.Mientras que no se encontraron relaciones de corte para las demás texturas, por este motivo se consideran desarrolladas en el mismo evento.

Parte de las texturas, como la pseudobandeada, pueden corresponder a sistema de baja sulfuración. Sin embargo, esta texturano muestra predominancia, por lo que este sistema podría estar parcialmente erodado.

La textura vuggy, no aflora, aunque es abundante en escombreras, lo que puede sugerir que haya sido extraído en la explotación de la veta o que aflore en las vetas cercanas.

La identificación de las fases más gruesas $(0,5-0,25 . \mathrm{mm})$ fue ajustada mediante calcografía (o microscopía de luz reflejada), lo que permitió redefinir la presencia de famatinita-luzonita, asociada a pirita, tenantita-tetraedrita, calcopirita, bismutinita y covelina.

A escala microscópica, en el mapa de metales, se observa una asociación metálica de As, $\mathrm{Cu}, \mathrm{Pb}, \mathrm{Sb}, \mathrm{Bi}$, Sn no identificada a escala de microscopio calcográfico y que se encontrarían en cristales de grano fino (menor a 10um). Este análisis realizado en un sector de una textura mineralizada en brechas, específicamente en clastos mineralizados, indica fases metálicas (como casiteritay sulfosal de $\mathrm{Bi}, \mathrm{Sb}, \mathrm{Pb}$ ?) de la primera etapa (Paaret al. 2010)junto con fases minerales (como bismutina y tetraedrita?) de segunda etapa (Paar et al. 2010). Así mismo, las muestras analizadas mediante microsonda electrónica muestran relación, principalmente, con el segundo evento y en menor medida con el primer eventode Paar et al. 2010 (Tabla

Tabla 2. Muestras estudiadas por microsonda electrónica y su asociación con el análisis textural, mineralógico y los eventos mineralizantes de Paar et al. (2010). Abreviaturas: Py: pirita, Calcpy: calcopirita, Bsm: bismutina, Fm: famatinita, Luz: luzonita, Cv: covellina.

\begin{tabular}{|l|l|l|l|}
\hline Muestras analizadas EPMA & Texturas & Asociación mineral & En relación antecedente Paar et al., (2010) \\
\hline E1D & vetillas & Py-Calcpy-Luz-Fm-Cv & 2do evento \\
\hline EV13B & brecha & Bsm-Py-calcpy-Fm & 2do evento con clastos de 1er evento \\
\hline Ev13A & brecha & Py-Calcpy-Fm-Cv & 2do evento con clastos de 1er evento \\
\hline E1B & pseudobandeada & Bsm-Py-Fm & 2do evento \\
\hline
\end{tabular}


2). No se ha distinguido texturas y mineralogía relacionada al tercer evento de Paar et al. (2010). Por esta razón se considera que sería necesario un estudio de detalle de la mineralización, a diferentes escalas, para definir la temporalidad de las texturas y la mineralización.

Las observaciones aportadas en este trabajo, tales como algunas texturas minerales junto con la presencia de minerales indicadores como luzonita-famatinita, sugerirían un epitermal de alta sulfuración (Sillitoe 1999, White 1991, Hedenquist et al., 1994, Hedenquist et al. 2000). Sin embargo, se destaca la importancia de realizar estudios sobre los depósitos vetiformes cercanos a la Veta Julio Verne para poder definir con precisión el modelo metalogenético y temporalidad de los eventos mineralizantes de la región «Depósito Organullo».

\section{CONCLUSIÓN}

Las texturas de mineralización observadas en la zona de Mina Julio Verne son pseudobandeadas, macivas, vetillas, brechosas, vuggy asociadas principalmente al segundo evento de estudios de referencias previos.

La mineralización de mena dada por, luzonita, famatinita, asociada a pirita, calcopirita, bismutinita y covelina, es típica de sistemas epitermales de alta sulfuración. Sin embargo se precisan estudios de mayor detalle en las vetas vecinas y de texturas y mineralización para determinar con precisión el modelo de mineralización.

\section{AGRADECIMIENTOS}

A financiamiento de estadías en el extranjero CONICET, a proyecto PIP Directora Dra. Cecilia del Papa. Al centro de instrumentación científica de Granada, España. A la técnica Isabel Guerra por su ayuda y colaboración. Al Instituto de Geocronología de Brasilia, Brasil. Los autores agradecen a los revisores Dres. Demartis y Montenegro, que han permitido enriquecer el manuscrito

\section{BIBLIOGRAFÍA}

Aceñolaza, F. y Aceñolaza, G. 2005. La Formación Puncoviscana y unidades estratigraficas vinculadas en el Neoproterozoico-Cambrico temprano del Noroeste Argentino. Latin American Journal of Sedimentology and basin analysis, 12 (2): 65-87.

Argañaraz, R. 1980. Proyecto Julio Verne. Informe Preliminar. Sociedad Minera Concordia SA, 3 pp. Salta.

Artha Resource Corporation, 2012. Argentina Organullo Gold Silver Property Update. Informe online, 7 pp. Canadá.

Blasco, G., Zappettini, E.O. y Hongn, F.D. 1996. Hoja Geológica 2566-II, San Antonio de los Cobres, 1:250.000. Secretaría de Minería de la Nación, Boletín 217, Buenos Aires.

Cardero Resource Coorperation Report 2011. Technical report on the Organullo Project. Vancouver, British Columbia, 37 pp. Canadá.

Coira, B. y Koukharsky, M. 1991. Lavas en almohadillas ordovícicas en el Cordón Escaya, Puna Septentrional, Argentina. Gto Congreso Geológico Chileno, Actas 1 (A-5): 674-678, Viña del Mar.

Coira, B. y Koukharsky, M. 1994. Complejos Submarinos Domos-Lávicos Silíceos de Edad Ordovícica en el Sector Oriental de la Puna Jujeña (22-234' sur]. Sus Implicancias. $7^{\circ}$ Congreso Geológico Chileno, Actas 2: 1000-1004, Concepción.

Coira, B. y Paris, G. 1981. Estratigrafía volcánica del área del cerro Tuzgle, Provincias de Jujuy y Salta. $8^{\circ}$ Congreso Geológico Argentino, Actas 3: 659-671, San Luis.

Fonseca, A. 2009. Organullo Project. Alteration mapping and Infrared Spectroscopy, Consultant report prepared for Cardero Resource Corp.

Gaines, R.V. 1957. Luzonite famatinite and some related minerals. The American Mineralogist, 42: 766-779.

Hayba, D.O., Bethke, P.M., Heald, P., Foley, N.K. 1985. Geologic, mineralogic and geochemical characteristics of volcanichosted epithermal precious-metal deposits: Reviews in Economic Geology, 2: 129-167.

Harrington, H. y Leanza, A. 1957. Ordovician trilobites of Argentina. Special Publication, Department of Geology. University of Kansas, Lawrence.

Heald, P., Foley, N.K. y Hayba, D.O. 1987. Comparative anatomy of volcanic-hosted epithermal deposits: acid-sulfate and 
adulariasericite types. Economic Geology 82 (1): 26 .

Hedenquist, J.W. 1987. Mineralization associated with volcanic-related hydrothermal systems in the Circum-Pacific Basin, in Transactions of the $4^{\circ}$ Circum Pacific Energy and Mineral Resources Conference, Singapore, 1986, Oklahoma, Circum Pacific Council for Energy and Mineral Resources, 1: 1-26.

Hedenquist, J.W., Arribas, A., y GonzalezUrien, E. 2000. Exploration for epithermal gold deposits: Reviews in Economic Geology, 13: 245-277.

Hedenquist, J.W., Matsuhisa, Y., Izawa, E., White, N.C., Giggenbach, W.F. y Aoki, M. 1994. Geology and geochemistry of highsulfidation Cu-Au mineralization in the Nansatsu district, Japan. Economic Geology 89 (1): 1-30.

Hidroproyectos, CETEC-CEPIC 1985. Estudios de la segunda fase de prefactibilidad geotérmica del área denominada Tuzgle, Departamento Susques. Ministerio de Economía, 488 pp. Provincia de Jujuy,

Japan International Cooperation Agency, 1993. Informe sobre la exploración de minerales del área oeste de la República Argentina, fase 1. Secretaría de Minería de la Nación, SEGEMAR, 167 pp., Buenos Aires.

Klipfel, P. 2009. Organullo Geological Report. Mineral Resource Services Inc. Reno, 38 pp. Estados Unidos.

Koukharsky, M. y Mirré, J.C. 1974. Nuevas evidencias de vulcanismo ordovícico en la Puna. Revista de la Asociación Geológica Argentina, 29: 128-134.

Omarini, R.H. 1983. Caracterización litológica, diferenciación y génesis de la Formación Puncoviscana entre el Valle de Lerma y la Faja Eruptiva de la Puna. Tesis Doctoral. Facultad de Ciencias Naturales, Universidad Nacional de Salta, 220 pp. Salta.

Paar, W.H, Sureda, R.J, Topa, D., y Brodtkorb, M.K. 2000 a. Los telururos de oro, krennerita, petzita y silvanita del prospecto Fátima, Distrito Minero Organullo, provincia de Salta. 5으 Congreso de Mineralogía y Metalogenia. Instituto de Recursos Minerales, Universidad Nacional de La Plata, Publicación 6: 369 373, La Plata.

Paar, W.H, Topa, D., de Brodtkorb, M.K y Sureda, R.J. 2000 b. Gladita PbCuBi5 S9, hodrushita Cu8 Bi12 S22, kawazulita Bi2SeTe2, krupkaita PbCuBi3S6, kuramita Cu3 SnS4, mohita Cu2 SnS3, vinciennita Cu10Fe4 Sn(As,Sb)S16, nue- vos minerales en la veta polimetalica de Julio Verne, Salta. 5ํ Congreso de Mineralogía y Metalogenia. Instituto de Recursos Minerales, Universidad Nacional de La Plata, Publicación 6: 374-380, La Plata.

Paar, W.H, Sureda, R.J. y Brodtkorb, M.K. de 2010. Nuevos datos de famatinitaluzonita y de enargita presentes en el distrito Julio Verne, Salta. 10 Congreso de Mineralogía y Metalogenia, Universidad Nacional de Río Cuarto. Edición electrónica, 367-368, Córdoba.

Petrinovic, I.A. 1989. Volcanismo Cenozoico asociado al lineamiento Calama-Olacapato-Toro en el tramo comprendido entre San Antonio de los Cobres y Olacapato, provincia de Salta, República Argentina. Informe Beca Doctoral CONICET, 108 pp. Salta.

Petrinovic, I.A., Mitjavila, J., Viramonte, J.G., Marti, J., Becchio, R., Arnosio, M. y Colombo, F. 1999. Descripción geoquímica y geocronológica de secuencias volcánicas neógenas de Trasarco, en el extremo oriental de la Cadena Volcánica Transversal del Quevar (Noroeste de Argentina). Acta Geológica Hispánica, 34 (2): 255-272.

Petrinovic, I.A., Marti, J., Aguirre Diaz, G.J., Guzmán, S., Geyer, A. y Salado Paz, N. 2010. The Cerro Aguas Calientes caldera, NW Argentina: an example of a tectonically controlled, polygenetic collapse caldera, and its regional significance. Journal of Volcanology and Geothermal Research 194 (1): 15-26.

Quiroga, M. 2014. Vulcanismo submarino del Paleozoico inferior, Quebrada del Cajón, Puna Salteña. Tesis Profesional. Universidad Nacional de Salta, 89 pp. Salta.

Ramos, V. 1973. Estructura de los primeros contrafuertes de la Puna Salto Jujeña y sus primeras manifestaciones volcánicas asociadas. $5^{\circ}$ Congreso Geológico Argentino, Actas 3: 354-385.

Ramallo, E., Becchio, R. y Bercheñi, V. 2011. Carta Minero-Metalogenética 2566-I, San Antonio de Los Cobres. Provincias de Salta y Jujuy. Instituto de Geología y Recursos Minerales, Servicio Geológico Minero Argentino, Boletín 394, 128 pp. Buenos Aires.

Rolleri, E.O. y Mingramm, A. 1968. Sobre el hallazgo del Ordovícico inferior al oeste de San Antonio de los Cobres (Provincia de Salta). Revista de la Asociación Geológica Argentina, 23 (2): 101-103

Sillitoe, R.H. 1999. Styles of high-sulphidation gold, silver and copper mineraliza- 
tion in the porphyry and epithermal environments. En in PacRim'99 Congress Proceedings, Bali, Indone.

Sureda, R.J., Paar, W.H. y Brodtkorb M.K. de 1994. Aikinita, benjaminita, emplectita, matildita y tetradimita en la paragénesis mineral bismutífera de la mina Julio Verne, provincia de Salta, Argentina. 7으 Congreso Geológico Chileno, Actas 2: 1229-1233, Concepción.

Torres Correjidor, P., Salado Paz, N., Da Silva Souza, V. y Floridia, A. 2016. Alteraciones hidrotermales en Organullo, mina Julio Verne, provincia de Salta. $11^{\circ}$ Congreso Argentino de Geología económica, Actas 1: 13. Salta.

Torres Correjidor, P. 2017. Caracterización de la alteración hidrotermal-mineralización de la Mina Julio Verne, depósito epitermal Organullo (Pb-Cu-Zn-Ag-Au), Puna central, provincia de Salta. Tesis profe- sional inédita. Facultad de Ciencias Naturales, Universidad Nacional de Salta, 180 pp. Salta

Turner, J.C.M. 1960. Estratigrafía de la Sierra de Santa Victoria y adyacencias. Boletín de la Academia Nacional de Ciencias 41 (2): 163-196, Córdoba.

Viera, C. 1975. Informe final del área de reserva nำ 26: Organullo, provincia de Salta. Centro de exploración geológico minera 2. Dirección General de Fabricaciones Militares. 47 pp. Salta.

White, N.C. 1991. High sulfidation epithermal gold deposits: Characteristics, and a model for their origin. En: Matsuhisa, Y., Aoki, M., y Hedenquist, J.W. (Eds) High-temperature Acid Fluids and Associated Alteration and Mineralization, Report No. 277: 9-20. Geological Survey of Japan, Japan. 